\title{
Coronary Arterial Blast After Blunt Politrauma, Case Report
}

\author{
Oscar Alonso Plaza* \\ National Institute of Legal Medicine and Forensic Sciences, Colombia
}

*Corresponding author: Oscar Alonso Plaza, National Institute of Legal Medicine and Forensic Sciences, Cali-Colombia, Faculty of Health Sciences, Pontificia Universidad Javeriana, Cali -Colombia, Faculty of Medicine, Santiago of Cali University, Cali-Colombia, Colombia

Submission: 悳 March 09, 2018; Published: 眥 September 20, 2018

\begin{abstract}
Background
We present a case report where a young adult who suffers a severe trauma with moderate cranial-brain trauma during a traffic accident, a closed trauma to the chest and abdomen, his general condition and what was observed during hospital admission, medical management is on track to cranial and abdominal traumas, despite surgical treatment dies. The post-mortem examination shows partial avulsion of pulmonary hilus and cardiac tamponade. During the revision of the heart, an oblique branch of the anterior descending artery with massive bleeding to the pericardial sac and subsequent cardiac tamponade as a basic cause of death is observed. The usual in cases of closed chest trauma with cardiac involvement is the laceration of the heart or coronary arteries by the displaced ribs, in this case the coronary burst was due to the sudden and exaggerated increase of the intrathoracic pressure due to the closed trauma without there being rib fractures.
\end{abstract}

Keywords: Politrauma; Coronary blast; Cardiac tamponade

\section{Introduction}

Traumatic lesions of vital organs at the thoracic level are a public health problem worldwide due to their progressive increase among young people and in most cases due to traffic accidents, large vessel involvement such as aortic artery and myocardial rupture are injuries quickly fatal. Maite A. Huis in Velt et al. [1] in her masterly work on Cardiac Trauma Revision clearly explains the types of cardiac injury and its pathophysiology [1], also in the available literature there are articles that expose that the closed chest trauma with coronary is associated to dissection and subsequent perforation with a vast series of ischemic, structural complications and heart rhythm disorders [2-7]. In the case report we expose the sequence of a closed chest trauma in a young adult without rib fractures and with burst-like rupture of a diagonal branch of the anterior descending coronary artery with subsequent cardiac tamponade and death.

\section{Case Presentation}

A young adult of 25 years old a motorcycle driver presents overturning with subsequent polytrauma and loss of consciousness for an undetermined time, enters the emergency room with amnesia of the event, Glasgow of 14/15, TA 122/61, FC 85x and 95\% Sat 02, due to the observed lesions, diagnostic approaches of moderate Traumatic Brain Injury, Cervical Trauma and Closed Trauma of the thorax and Trauma without hemodynamic instability and hepatic trauma to be discarded are made, the most evident to the examination are parallel abrasions in the left pectoral region. The chest $\mathrm{X}$-rays do not show rib fractures, normal cardiac silhouette without evidence of hemothorax or pneumothorax. A brain CT scan shows large cerebral edema and a CT scan of the thoracic spine shows vertebral body fractures without spinal cord involvement. It is assessed by a neurosurgeon who orders anti-edema measures and reassessment and is examined by a surgeon who orders surgery for mini-laparotomy. Discard splenic or hepatic trauma Nine hours after admission presents severe abdominal pain with tachycardia and hypotension, there is poor response of hemodynamic stabilization. Due to the abdominal symptomatology, a laparotomy is performed where 1000cc of saline fluid is found at the peripancreatic level and post-trauma pancreatitis. During the intervention, the patient presents hemodynamic instability, the patient goes out in poor surgical conditions, after two cardiac arrests. Respiratory is declared dead. He died 13 hours after admission to the hospital.

The post-mortem examination shows parallel abrasions on left pectoral region, open surgical wound in the middle line of the abdomen; internally there are hematomas that infiltrate pectoral muscles, there are no rib fractures, there are subpleural hematomas with partial avulsion of pulmonary hilus, there is bilateral hemothorax of $600 \mathrm{cc}$, hemopericardium at tension of $120 \mathrm{cc}$ approx. (Figure 1). There is an open surgical wound in the midline 
of the abdomen with edematous pancreas at the level of the neck and body. Careful examination of the heart shows an area of cardiac contusion involving the anterior aspect of the two ventricles and a burst-like laceration of an oblique branch of the anterior descending artery with the shape of an external crater (Figure 2).

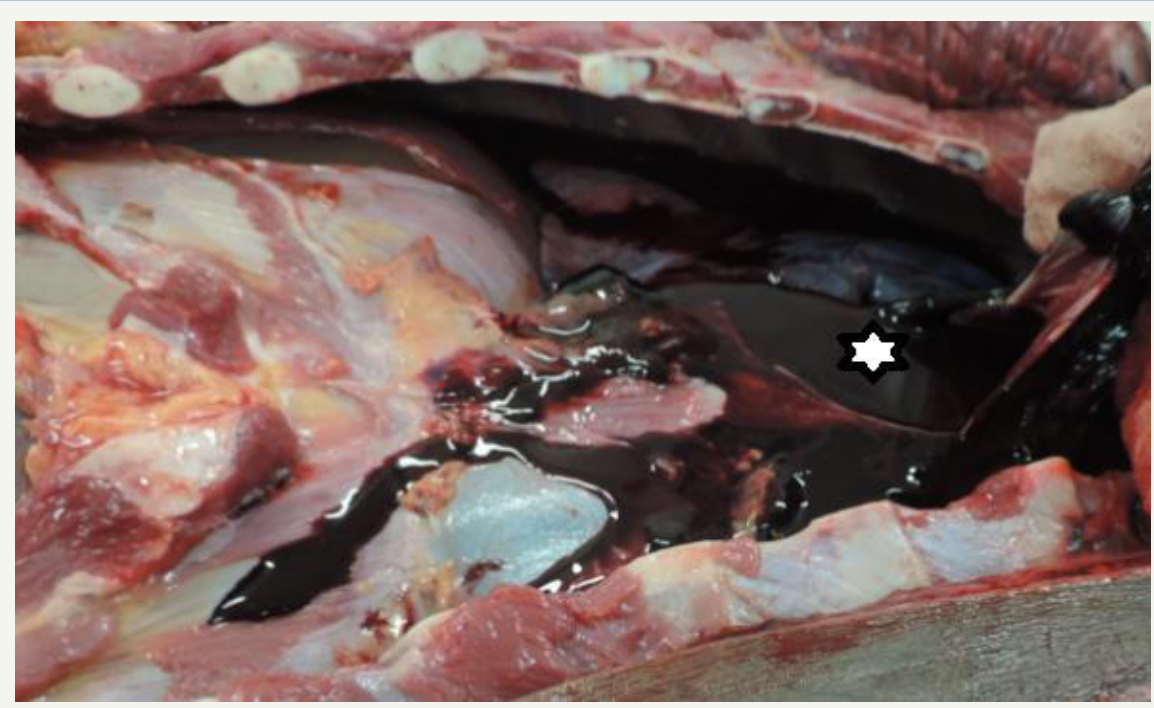

Figure 1: The white star shows the hemopericardium.

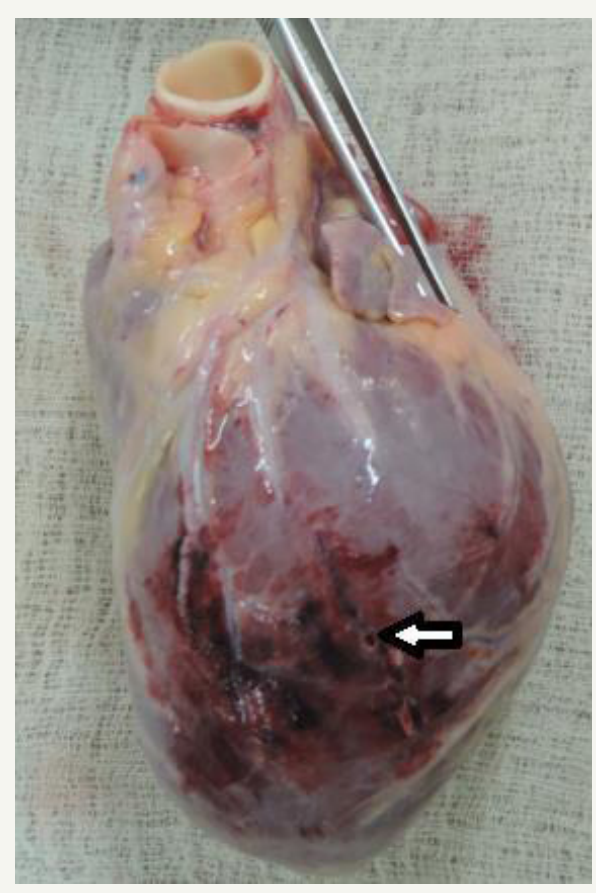

Figure 2: The white arrow indicates the coronary burst.

\section{Discussion}

The heart trauma in any presentation, whether in fights, falls of heights, sporting events and/or traffic accident will generate a high morbidity and high mortality by including in order of severity of presentation a cordio cordis, myocardial contusions, acute myocardial infarction and tissue disrupted lesions such as rupture of the pericardial, myocardial, valvular and septal sac. In reviewing the existing literature, it was found that isolated coronary ruptures are necropsy findings and are accompanied by myocardial contu- sions due to rib fractures that perforate the myocardium and/ or coronary artery, for which case there were no rib fractures and the burst was due to a momentary and marked increase in intrathoracic pressure during the traffic accident that caused the bursting of this branch of the coronary artery. The rupture of this ramification of the coronary artery produced constant and permanent bleeding into the pericardial sac with the tamponade leading to respiratory failure and subsequent death while the patient presented more severe signs and symptoms due to the involvement of other organs. 
The assessment of a patient of a polytraumatized patient with thoracic involvement is not only focused on the determination of lesions by means such as RX and/ or CAT, other paraclinical ones should be taken such as EKG, echocardiogram and cardiac enzymes, given that some lesions cardiac and coronary arteries will be masked by the associated traumas, for the case report the careful observation and the diagnostic suspicion with the accomplishment of the paraclinical mentioned above could have improved the prognosis of the deceased.

\section{Conclusion}

Coronary perforations require early diagnosis and open surgical management or by stenting, we present this case where the lesion occurs in a small branch of the anterior descending artery and with a burst type pattern not associated with rib fractures due to a transient increase of intra-thoracic pressure in a traffic accident which generated progressive bleeding until cardiac tamponade. The complications of polytrauma are unavoidable despite the skill and preparation of the doctor, therefore it is a duty to try to minimize the possibility of aggravation by using all available therapeutic arsenal recognizing and managing the myriad of possible compli- cations in a patient who does not It improves after an opportune treatment.

\section{References}

1. Huis In 't Veld MA, Craft CA, Hood RE (2018) Blunt Cardiac Trauma Review. Cardiol Clin 36(1): 183-191.

2. Maheshwari A, Thenappan T, Das G (2017) Adding fuel to the fire: Coronary artery dissection complicating blunt chest trauma. American Journal of Emergency Medicine 35(7): 1041.e5-1041.e6.

3. Adler JD, Scalea TM (2010) Right coronary artery dissection after blunt chest trauma. Injury Extra 41: 77-79.

4. Heyndrlckx G, Vermeire P, Goffin Y, Van den Bogaert P (1974) Rupture of the right coronary artery due to nonpenetrating chest trauma. Chest 65(5): 577-579.

5. Himanshu Mahla RR, Shivanand P, Kasam Subramanyam S, Manjunath NC (2014) Coronary perforation with cardiac tamponade - Bailed out with the covered stent. Journal of Indian College of Cardiology 4(3): 194196.

6. Reiss J, Razzouk AJ, Jon K, Ramesh B, Leonard LB (2001) Concomitant traumatic coronary artery and tricuspid valve injury: a heterogeneous presentation. J Trauma 50(5): 942-944.

7. Suzuki I, Sato M, Hoshi N, Hiroshi N (2000) Coronary arterial laceration after blunt chest trauma. N Engl J Med 343: 742-743.
Creative Commons Attribution 4.0 International License

For possible submissions Click Here

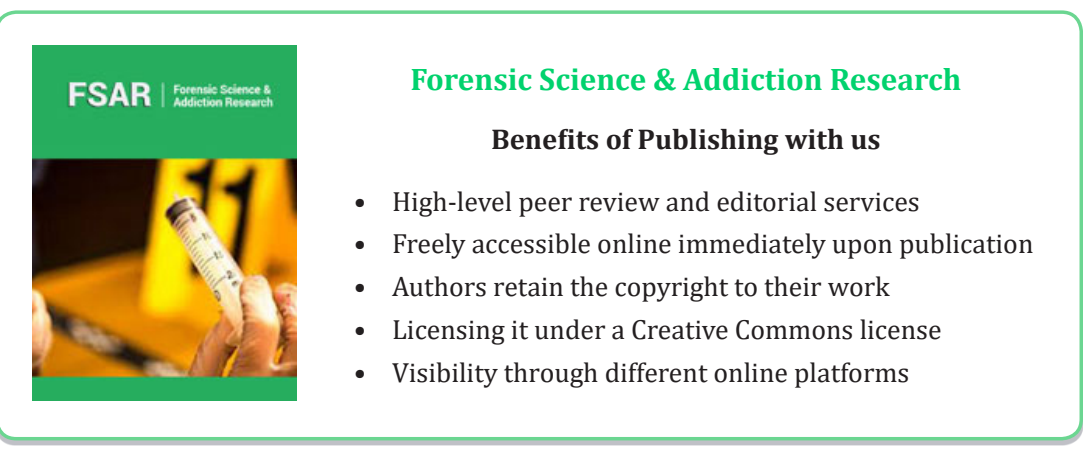

